\title{
INVESTIGATING THE MINIMUM ENERGY PRINCIPLE IN SEARCHES FOR NEW MOLECULAR SPECIES-THE CASE OF $\mathrm{H}_{2} \mathrm{C}_{3} \mathrm{O}$ ISOMERS
}

\author{
Ryan A. Loomis ${ }^{1,8}$, Brett A. McGuire ${ }^{2,3,9}$, Christopher Shingledecker ${ }^{4}$, Chelen H. Johnson ${ }^{5,10}$, \\ Samantha Blair ${ }^{6}$, Amy Robertson ${ }^{7}, 11$, and Anthony J. RemiJan ${ }^{2}$ \\ ${ }^{1}$ Department of Astronomy, Harvard University, Cambridge, MA 02138, USA; rloomis@cfa.harvard.edu \\ 2 National Radio Astronomy Observatory, Charlottesville, VA 22904, USA \\ ${ }^{3}$ Division of Chemistry and Chemical Engineering, California Institute of Technology, Pasadena, CA 91125, USA \\ ${ }^{4}$ Department of Astronomy, University of Virginia, Charlottesville, VA 22904, USA \\ 5 Breck School, Golden Valley, MN 55422, USA \\ ${ }^{6}$ Department of Natural Sciences, Dalton State, Dalton, GA 30720, USA \\ ${ }^{7}$ Department of Astronomy, University of Arizona, Tucson, AZ 85721, USA \\ Received 2014 August 22; accepted 2014 October 30; published 2015 January 14
}

\begin{abstract}
Recently, Lattelais et al. have interpreted aggregated observations of molecular isomers to suggest that there exists a "minimum energy principle," such that molecular formation will favor more stable molecular isomers for thermodynamic reasons. To test the predictive power of this principle, we have fully characterized the spectra of the three isomers of $\mathrm{C}_{3} \mathrm{H}_{2} \mathrm{O}$ toward the well-known molecular region Sgr B2(N). Evidence for the detection of the isomers cyclopropenone $\left(\mathrm{c}-\mathrm{C}_{3} \mathrm{H}_{2} \mathrm{O}\right)$ and propynal $(\mathrm{HCCCHO})$ is presented, along with evidence for the non-detection of the lowest zero-point energy isomer, propadienone $\left(\mathrm{CH}_{2} \mathrm{CCO}\right)$. We interpret these observations as evidence that chemical formation pathways, which may be under kinetic control, have a more pronounced effect on final isomer abundances than thermodynamic effects such as the minimum energy principle.
\end{abstract}

Key words: astrochemistry - ISM: abundances - ISM: clouds - ISM: molecules - molecular processes

\section{INTRODUCTION}

A long-standing goal of astrochemistry is to understand the physical and chemical evolution of interstellar sources through molecular observations. The formation and destruction of complex organic molecules are affected by the source conditions; observed molecular abundance ratios preserve information about the physical evolution of the region, acting as a chemical fingerprint. Extracting this information has proven to be exceedingly difficult, however, for even the simplest sources. Due to complex source structure, incomplete molecular inventories of sources, vast networks of molecular interactions, and only a partial understanding of interstellar chemical formation pathways, it is challenging to create models sufficiently accurate to predict observations (Quan \& Herbst 2007).

Numerous efforts have sought to address these issues by establishing complete molecular inventories for molecule-rich sources and characterizing basic conditions and abundances (e.g., Neill et al. 2014; Crockett et al. 2014), and creating complete reaction networks with accurate reaction dynamics (e.g., Garrod et al. 2008; Garrod 2013). Still, the low densities, low temperatures, and long timescales of molecular processes in the interstellar medium (ISM) present a significant challenge to relating laboratory reaction dynamics and mechanisms to those occurring in the ISM (Laas et al. 2013). Whether the reactions governing the formation of complex molecules are primarily under kinetic or thermodynamic control is of particular interest, as this significantly affects molecular abundance ratios

\footnotetext{
8 R. A. Loomis was a summer student at the National Radio Astronomy Observatory.

9 B. A. McGuire is a Jansky Fellow of the National Radio Astronomy Observatory.

${ }^{10} \mathrm{C}$. H. Johnson was a participant in the RET program at the National Radio Astronomy Observatory.

${ }^{11}$ A. Robertson was a summer student at the National Radio Astronomy Observatory.
}

and the formation of prebiotically relevant molecules (Garrod et al. 2008; Lovas et al. 2010).

Observations of molecular isomers provide a unique tool for directly probing hypotheses about reaction mechanisms in the ISM, as their formation and destruction routes directly influence their abundance ratios (Hollis et al. 2006). While this is true for all molecules, isomers are particularly useful as they present a similar level of chemical complexity, and have the same constituent atoms, simplifying the problem tremendously. This approach has historically proven to be fruitful; hypotheses of the formation chemistry of $\mathrm{HCN}$ led to the detection of its isomer, HNC (Snyder \& Buhl 1971), and comparisons between the abundances and distributions of conformational isomers of methyl formate, ethanol, and vinyl alcohol have yielded important information about their formation pathways (Neill et al. 2012; Pearson et al. 1997; Turner \& Apponi 2001). Thus, understanding the root causes of molecular abundance ratios and accurately predicting which isomers are most likely to be observed is a highly desirable goal. Comparing abundance ratios with the conversion barriers and zero-point energy differences between the different isomers can elucidate whether a thermal process or a kinetically controlled formation mechanism is primarily responsible in determining the ratio.

Recently, (Lattelais et al. 2009, 2010, 2011) investigated the relative abundances of 32 detected molecular isomers and suggested that there exists a "minimum energy principle" (MEP), generally applicable across a number of astronomical environments. According to this principle, the isomer with the lowest zero-point bonding energy (i.e., the most energetically stable isomer) should be the most abundant, and thus most easily detected. Lattelais et al. (2009) include a wide variety of sources in this analysis, including molecular clouds, hot cores/corinos, photodissociation regions, and asymptotic giant branch stars, and do not note any differences in trends between these sources. Importantly, they make no mention of attempting to exclude 


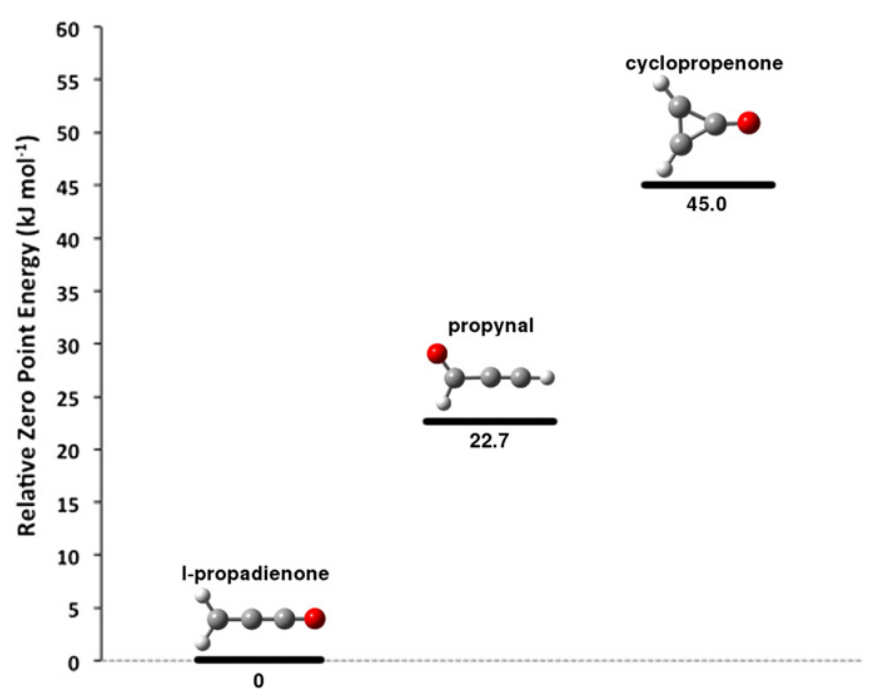

Figure 1. Relative binding energies of the three $\mathrm{C}_{3} \mathrm{H}_{2} \mathrm{O}$ isomers.

sources where LTE assumptions would be unlikely to hold, and instead assert that LTE effects may be general across all sources used in their analysis. $\mathrm{C}_{4} \mathrm{H}_{3} \mathrm{~N}$ and $\mathrm{C}_{2} \mathrm{H}_{4} \mathrm{O}_{2}$ are noted as exceptions to the principle, but are dismissed by calling the abundance measurements of the former into question and proposing differential adsorption rates onto grains for the latter (Lattelais et al. 2009, 2010). However, studies of other common isomers (e.g., HCN/HNC) have shown that abundances and emission strength are dependent on the local environment, suggesting that there must be more to the picture than bonding energy ratios (see, e.g., Sarrasin et al. 2010, and references therein). We therefore assert that the chemical formation route and physical conditions of these species, especially complex organics, are more significant in establishing relative abundance ratios than the zero-point bonding energies, and thus the MEP is not generally applicable as proposed by Lattelais et al. (2009, 2010, 2011).

Toward this end, we have fully characterized the spectra of the three isomers of $\mathrm{C}_{3} \mathrm{H}_{2} \mathrm{O}$ toward the well-studied molecular region $\mathrm{Sgr} \mathrm{B} 2(\mathrm{~N})$. The relative bonding energies of these isomers are shown in Figure 1. Based on these bonding energies and with no other prior knowledge, the MEP would predict the most stable isomer, propadienone $\left(\mathrm{CH}_{2} \mathrm{CCO}\right)$, to be observed as the most abundant of the three isomers (Karton \& Talbi 2014). However, propadienone has never been detected toward an astronomical source, while both propynal and cyclopropenone have been detected in molecular clouds (Irvine et al. 1988; Hollis et al. 2006). We present evidence for the detection of the two higher energy isomers cyclopropenone $\left(c-\mathrm{C}_{3} \mathrm{H}_{2} \mathrm{O}\right)$ and propynal (HCCCHO), along with evidence of the nondetection of propadienone toward Sgr B2(N). We interpret this to show that chemical formation pathways, which may be under kinetic control, have a more pronounced effect on final isomer abundances than thermodynamic effects such as the MEP.

\section{OBSERVATIONS}

Sgr B2(N) is one of the preeminent sources for complex molecule detections, and the majority of all molecules used in the analysis of Lattelais et al. (2009) have been detected there, thus it serves as a reasonable source to test the predictions of the MEP. Sgr B2(N) has a heterogeneous temperature and density structure, with both a hot-core and a colder, less dense envelope, yielding multiple velocity components and a complex chemistry (Belloche et al. 2013). For a full discussion of the effect of this structure on molecular inventories of the respective regions, see Neill et al. (2014). Significantly, many of the large complex organic molecules observed toward Sgr B2(N) (including some traditionally considered to be hot-core molecules) have been seen to have sub-thermal emission, with significant non-LTE effects (Neill et al. 2012; Loomis et al. 2013; Zaleski et al. 2013, and references therein). These molecules have very low rotational temperatures, suggesting that they are extended and belong to the envelope, but the non-LTE effects make determinations of column density and temperature extremely difficult without collisional cross-sections (Faure et al. 2014). As all three of the molecules searched for in this study would be expected to originate in colder environments, these non-LTE effects are likely very relevant, and are discussed in Section 4.1.

All known strong (predicted to be above the measured noise limit at a characteristic excitation temperature of $10-50 \mathrm{~K}$ ) rotational transitions of cyclopropenone, propynal, and propadienone between $4 \mathrm{GHz}$ and $50 \mathrm{GHz}$ were searched for in publicly available data from the PRIMOS survey of Sgr B2(N), a NRAO key project on the Robert C. Byrd Green Bank Telescope (GBT) from 2008 January through 2011 July. ${ }^{12}$ An LSR source velocity of $+64 \mathrm{~km} \mathrm{~s}^{-1}$ was assumed, and antenna temperatures were recorded on the $T_{A}^{*}$ scale (Ulich \& Haas 1976) with estimated $20 \%$ uncertainties. Data were taken in positionswitching mode, with 2 minute scans toward the on position $\left(\alpha \mathrm{J} 2000=17^{\mathrm{h}} 47^{\mathrm{m}} 19^{\mathrm{s}} .8, \delta \mathrm{J} 2000=-28^{\circ} 22^{\prime} 17^{\prime \prime} .0\right)$ and toward the off position, $1^{\circ}$ east in azimuth. Additional details of PRIMOS survey observations can be found in Neill et al. (2012). Rest frequencies for all transitions were obtained from the Splatalogue database. ${ }^{13,14}$

\section{RESULTS}

\subsection{Cyclopropenone}

Nine low-energy, high line-strength transitions of cyclopropenone were identified in absorption in PRIMOS data. Of these, six had been previously identified by Hollis et al. (2006) at lower sensitivity and spectral resolution (see Table 1). All observed transitions were $a$-type $\left(\mu_{a}=4.39(06) \mathrm{D}\right)$ (Benson et al. 1973), with six $R$-branch transitions $(\Delta J=-1)$ and three $Q$-branch $(\Delta J=0)$. Three higher energy $(J>4) Q$-branch transitions were not observed. This encompasses all strong transitions within the PRIMOS frequency coverage, with the exception of the $2_{12}-1_{11}$ transition where observational data were not available.

All detected transitions were observed at a nominal rest velocity of $v_{\mathrm{LSR}}=+64 \mathrm{~km} \mathrm{~s}^{-1}$, with a second, weaker, $v_{\mathrm{LSR}}=$ $+82 \mathrm{~km} \mathrm{~s}^{-1}$ component observed for all transitions other than $1_{01}-0_{00}$; consistent with other molecular detections with the GBT toward Sgr B2(N) (see, e.g., Hollis et al. 2004a; Remijan et al. 2008, and references therein). Observations of all $Q$ - and $R$-branch transition rest frequencies within the PRIMOS coverage are presented in Figures 2 and 3, respectively.

\footnotetext{
12 Access to the entire PRIMOS data set and specifics on the observing strategy including the overall frequency coverage, are available at http://www.cv.nrao.edu/ aremijan/PRIMOS/.

13 Original laboratory data for PRIMOS frequency ranges are from Benson et al. (1973), Winnewisser (1973), and Brown et al. (1987). Cyclopropenone and propadienone frequencies are cataloged through CDMS (Müller et al. 2001, 2005), and propynal through JPL (Pickett et al. 1998).

14 Available at www.splatalogue.net (Remijan et al. 2007).
} 
Table 1

Observed Transitions of Cyclopropenone

\begin{tabular}{|c|c|c|c|c|c|c|c|}
\hline \multirow{2}{*}{$\begin{array}{l}\text { Transition } \\
\mathrm{J}_{k k}^{\prime}-\mathrm{J}_{k k}^{\prime \prime}\end{array}$} & \multirow{2}{*}{$\begin{array}{c}\text { Frequency }{ }^{\mathrm{a}, \mathrm{b}} \\
(\mathrm{MHz})\end{array}$} & \multirow{2}{*}{$\begin{array}{l}E_{u} \\
(\mathrm{~K})\end{array}$} & \multirow{2}{*}{$\begin{array}{l}S_{i j} \mu^{2} \\
\left(\mathrm{D}^{2}\right)\end{array}$} & \multicolumn{2}{|c|}{$64 \mathrm{~km} \mathrm{~s}^{-1}$} & \multicolumn{2}{|c|}{$82 \mathrm{~km} \mathrm{~s}^{-1}$} \\
\hline & & & & $\Delta T_{A}^{* \mathrm{c}}(\mathrm{mK})$ & $\Delta V^{\mathrm{c}}\left(\mathrm{km} \mathrm{s}^{-1}\right)$ & $\Delta T_{A}^{* \mathrm{c}}(\mathrm{mK})$ & $\Delta V^{\mathrm{c}}\left(\mathrm{km} \mathrm{s}^{-1}\right)$ \\
\hline $2{ }_{11}-2_{12}$ & 4632.79 & 3.34 & 48.19 & $-57.2(5.4)$ & $14.8(0.9)$ & $-32.1(4.6)$ & $15.1(1.2)$ \\
\hline $3_{12}-3_{13} d$ & 9263.49 & 5.48 & 33.76 & $-29.1(6.1)$ & $15.9(0.5)$ & $-10.0(0.9)$ & $12.4(1.1)$ \\
\hline $4_{13}-4_{14} d$ & 15427.90 & 8.33 & 26.12 & $-12.1(0.6)$ & $11.6(0.6)$ & $-5.4(0.7)$ & $12.0(0.8)$ \\
\hline $5_{14}-5_{15}$ & 23105.58 & 11.88 & 21.41 & $<5.0$ & $\ldots$ & $<5.0$ & $\ldots$ \\
\hline $6_{15}-6_{16}$ & 32255.63 & 16.14 & 18.27 & $<10.7$ & $\ldots$ & $<10.7$ & $\ldots$ \\
\hline $7_{16}-7_{17}$ & 42808.10 & 21.08 & 16.10 & $<7.3$ & $\ldots$ & $<7.3$ & $\cdots$ \\
\hline $1_{01}-0_{00}{ }^{d}$ & 14105.74 & 0.68 & 19.27 & $-6.7(0.7)$ & $7.2(0.9)$ & $<2.2$ & $\ldots$ \\
\hline $2_{12}-1_{11}$ & 26667.02 & 3.12 & 86.73 & $\ldots$ & $\ldots$ & $\ldots$ & $\ldots$ \\
\hline $2_{02}-1_{01}$ & 28139.89 & 2.03 & 38.52 & $-133.6(4.2)$ & $8.6(0.3)$ & $-48.9(4.5)$ & $6.4(0.5)$ \\
\hline $2{ }_{11}-1_{10}$ & 29755.51 & 3.34 & 86.73 & $-83.2(8.8)$ & $8.2(0.5)$ & $-22.9(7.3)$ & $14.2(2.4)$ \\
\hline $3_{13}-2_{12}{ }^{\mathrm{d}}$ & 39956.70 & 5.04 & 154.15 & $-66.5(3.1)$ & $6.0(0.3)$ & $-32.9(3.0)$ & $5.9(0.6)$ \\
\hline $3_{03}-2_{02}{ }^{\mathrm{d}}$ & 42031.94 & 4.04 & 57.71 & $-74.5(1.9)$ & $15.3(0.4)$ & $-40.3(2.2)$ & $9.4(0.5)$ \\
\hline $3_{12}-2_{11}^{d}$ & 44587.40 & 5.48 & 154.1 & $-46.9(2.1)$ & $11.7(0.6)$ & $-34.4(1.8)$ & $12.5(0.9)$ \\
\hline
\end{tabular}

Notes.

${ }^{a}$ Beam sizes, efficiencies, and continuum temperatures for each respective frequency can be found in Hollis et al. (2007).

${ }^{\mathrm{b}}$ Rest frequencies are all taken from Splatalogue; see Section 2 for complete references.

$\mathrm{c}$ The uncertainties for the intensities and line widths are type $\mathrm{B}, k=1(1 \sigma)$ (Taylor \& Kuyatt 1994).

${ }^{\mathrm{d}}$ Previously detected with the GBT with lower sensitivity and spectral resolution (Hollis et al. 2006).

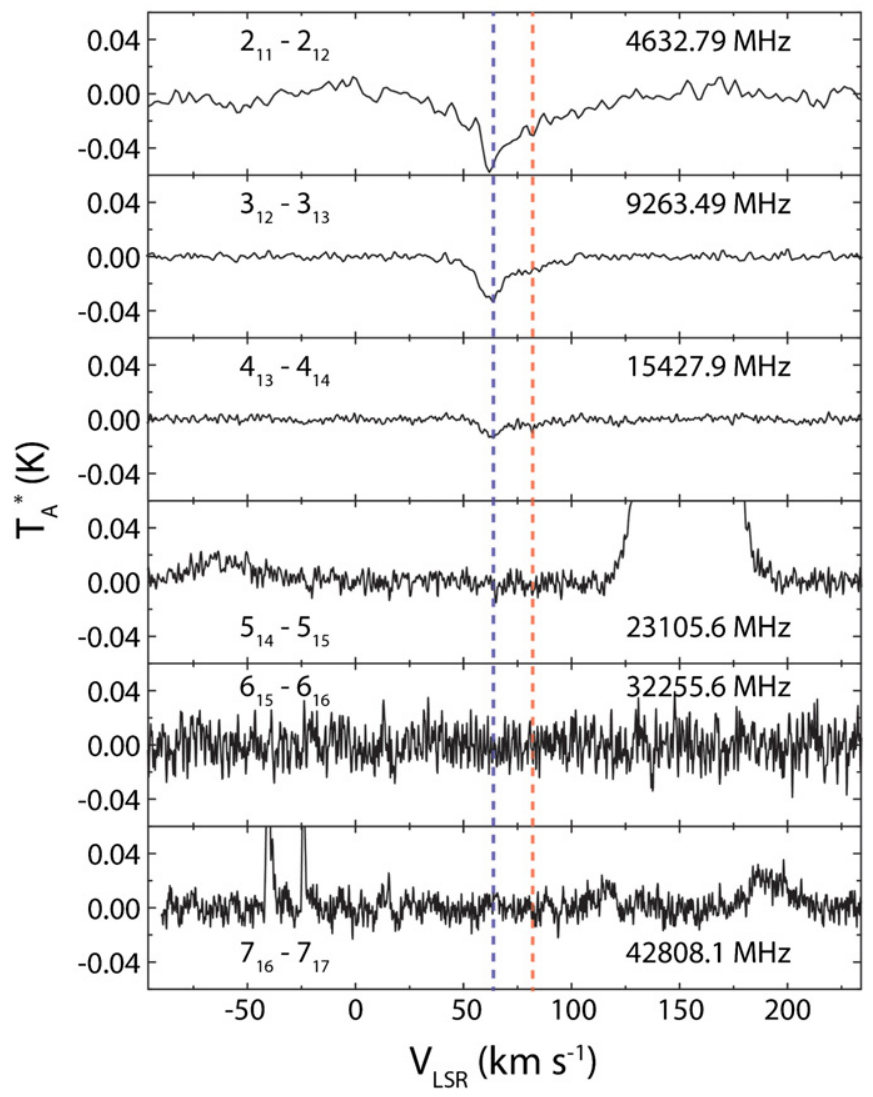

Figure 2. Observed cyclopropenone $Q$-branch transitions in PRIMOS data toward Sgr B2(N). Dashed vertical lines indicate the primary $+64 \mathrm{~km} \mathrm{~s}^{-1}$ component (blue) and the secondary $+82 \mathrm{~km} \mathrm{~s}^{-1}$ component (red).

As seen in Figure 3, the $2_{11}-1_{10}$ transition is blended with a hydrogen recombination line $(\mathrm{H}(94) \delta)$ at $29575.189 \mathrm{MHz}$. Additionally, the $v_{\mathrm{LSR}}=+64 \mathrm{~km} \mathrm{~s}^{-1}$ component of the $3_{13}-2_{12}$ transition is partially blended with the $2_{02}-1_{01}, v=1$ transition of $\mathrm{NH}_{2} \mathrm{CN}$ in emission. Best-fit Gaussian line widths and peak intensities, or respective upper limits, are reported in Table 1.
The $2_{11}-1_{10}$ transition was simultaneously fit in absorption against a modeled Gaussian emission profile for the $\mathrm{H}(94) \delta$ recombination line.

\subsection{Propynal}

Six uncontaminated, low-energy, high line-strength transitions of propynal were identified in absorption. One of these had been previously been detected by Hollis et al. (2004b) with lower sensitivity and spectral resolution (see Table 2). Three of the identified transitions were $\mathrm{k}_{a}=0 a$-type $\left(\mu_{a}=2.359(18) \mathrm{D}\right)$, and three were $b$-type $\left(\mu_{b}=1.48(22) \mathrm{D}\right)$ (Brown \& Godfrey 1984). Of the remaining twelve low-energy, high line-strength transitions predicted to be possibly observable, six were not observed above the noise level, five were contaminated by blends with other molecule emission features (see Figure 4), and frequency coverage was not available for one transition. Notably, although multiple $\mathrm{k}_{a}=0 a$-type transitions were observed with strong absorption, no $k_{a}=1 a$-type transitions were detected.

Irvine et al. (1988) suggested that the $k_{a}=1$ transitions of propynal may be weaker than predicted by a thermal model due to $b$-type transitions transferring $k_{a}=1$ population into the $k_{a}=0$ state; this behavior has also been observed in HNCO (Hocking et al. 1974). Irvine et al. used a collisional model to predict corrected $k_{a}=1$ intensities for their detection in TMC-1, and determined an approximate intensity in agreement with their tentative $2 \sigma-3 \sigma$ detection of the $2_{11}-1_{10}$ transition using the NRAO $140 \mathrm{ft}$. If this model is correct, a rough scaling to our observations would explain the lack of $k_{a}=1$ detections, with all predicted intensities below the observed noise level.

All detected transitions were observed at a nominal rest velocity of $v_{\mathrm{LSR}}=+64 \mathrm{~km} \mathrm{~s}^{-1}$, with an additional, weaker, $v_{\text {LSR }}=+82 \mathrm{~km} \mathrm{~s}^{-1}$ component observed for all transitions other than $4_{04}-3_{03}$, where the noise level is comparable to the expected intensity of the $v_{\mathrm{LSR}}=+82 \mathrm{~km} \mathrm{~s}^{-1}$ component. Observations of all $a$-type and $b$-type transition rest frequencies within PRIMOS coverage are presented in Figures 4 and 5, respectively. Best-fit Gaussian line widths and peak intensities, or respective upper limits, are reported in Table 2. All features were observed with line widths of $\sim 4-10 \mathrm{~km} \mathrm{~s}^{-1}$ with the exception of the $5_{15}-6_{06}$ 


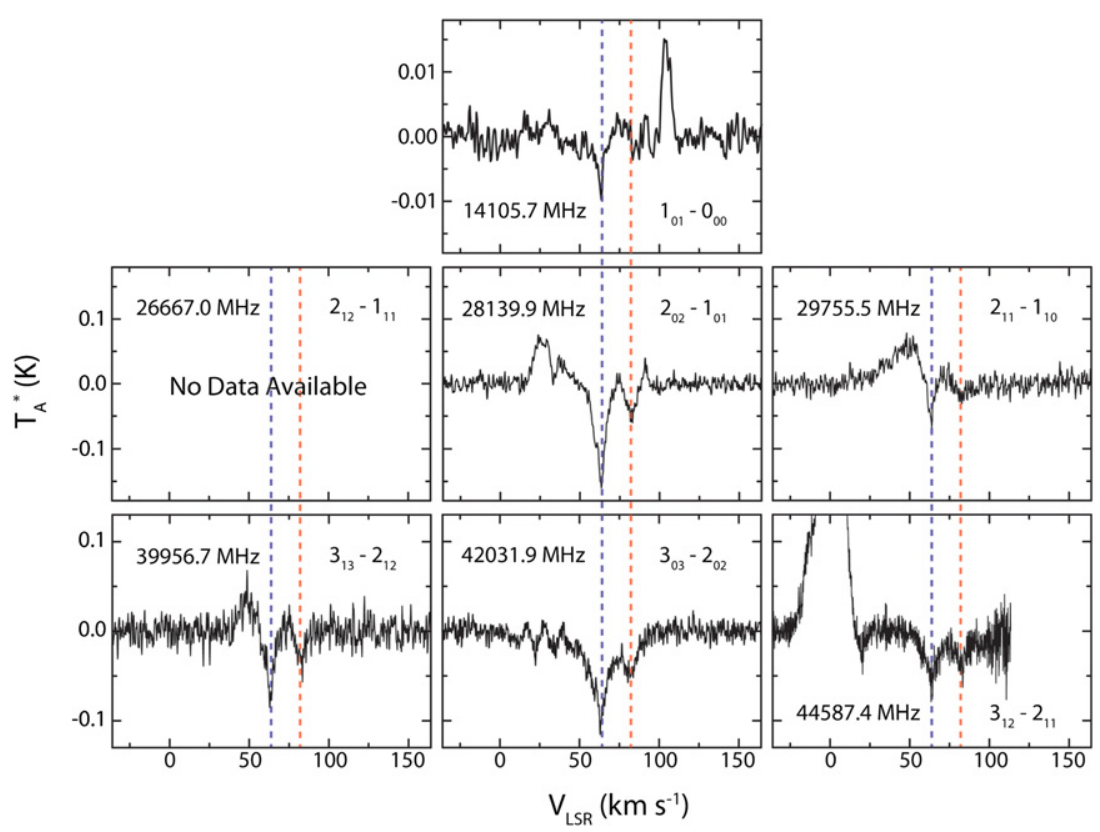

Figure 3. Observed cyclopropenone $R$-branch transitions in PRIMOS data toward $\mathrm{Sgr}$ B2(N). Dashed vertical lines indicate the primary $+64 \mathrm{~km} \mathrm{~s}^{-1}$ component $(\mathrm{blue})$ and the secondary $+82 \mathrm{~km} \mathrm{~s}^{-1}$ component (red).

Table 2

Observed Transitions of Propynal

\begin{tabular}{|c|c|c|c|c|c|c|c|}
\hline \multirow{2}{*}{$\begin{array}{l}\text { Transition } \\
J_{k k}^{\prime}-J_{k k}^{\prime \prime}\end{array}$} & \multirow{2}{*}{$\begin{array}{c}\text { Frequency }{ }^{\mathrm{a}, \mathrm{b}} \\
(\mathrm{MHz})\end{array}$} & \multirow{2}{*}{$\begin{array}{l}E_{u} \\
(\mathrm{~K})\end{array}$} & \multirow{2}{*}{$\begin{array}{l}S_{i j} \mu^{2} \\
\left(\mathrm{D}^{2}\right)\end{array}$} & \multicolumn{2}{|c|}{$64 \mathrm{~km} \mathrm{~s}^{-1}$} & \multicolumn{2}{|c|}{$82 \mathrm{~km} \mathrm{~s}^{-1}$} \\
\hline & & & & $\Delta T_{A}^{* \mathrm{c}}(\mathrm{mK})$ & $\Delta V^{\mathrm{c}}\left(\mathrm{km} \mathrm{s}^{-1}\right)$ & $\Delta T_{A}^{* \mathrm{c}}(\mathrm{mK})$ & $\Delta V^{\mathrm{c}}\left(\mathrm{km} \mathrm{s}^{-1}\right)$ \\
\hline $5_{15}-6_{06}$ & 5044.66 & 9.64 & 5.58 & $-36.4(2.2)$ & $20.3(1.8)^{\mathrm{d}}$ & $-26.1(2.3)$ & $20.3(1.8)^{\mathrm{d}}$ \\
\hline $4_{14}-5_{05}{ }^{\mathrm{e}}$ & 15146.04 & 7.44 & 4.43 & $-20.3(1.2)$ & $9.9(0.6)$ & $-14.5(1.3)$ & $10.3(0.9)$ \\
\hline $3_{13}-4_{04}$ & 25100.65 & 5.68 & 3.30 & $-20.4(1.5)$ & $10.1(0.8)$ & $-16.6(1.6)$ & $8.3(0.9)$ \\
\hline $2_{12}-3_{03}$ & 34903.64 & 4.36 & 2.18 & $<8.7$ & $\ldots$ & $<8.7$ & $\ldots$ \\
\hline $1_{11}-2_{02}$ & 44550.03 & 3.48 & 1.09 & $<8.6$ & $\ldots$ & $<8.6$ & $\ldots$ \\
\hline $1_{01}-0_{00}$ & 9325.81 & 0.45 & 5.56 & $<3.8$ & $\ldots$ & $<3.8$ & $\ldots$ \\
\hline $2_{12}-1_{11}$ & 18325.56 & 4.36 & 8.35 & Blend & $\ldots$ & Blend & $\ldots$ \\
\hline $2_{02}-1_{01}$ & 18650.31 & 1.34 & 11.13 & $-16.8(1.6)$ & $7.2(0.7)$ & $-14.1(1.5)$ & $4.9(0.6)$ \\
\hline $2_{11}-1_{10}$ & 18978.79 & 4.41 & 8.35 & $<3.6$ & $\ldots$ & $<3.6$ & $\ldots$ \\
\hline $3_{13}-2_{12}$ & 27487.45 & 5.68 & 14.84 & Blend & $\ldots$ & $<8.3$ & $\ldots$ \\
\hline $3_{03}-2_{02}$ & 27972.13 & 2.68 & 16.69 & Blend & $\ldots$ & Blend & $\ldots$ \\
\hline $3_{12}-2_{11}$ & 28467.15 & 5.77 & 14.84 & $\ldots$ & $\ldots$ & $\ldots$ & $\ldots$ \\
\hline $4_{14}-3_{13}$ & 36648.20 & 7.44 & 20.87 & $<11.2$ & $\ldots$ & $<11.2$ & $\ldots$ \\
\hline $4_{04}-3_{03}$ & 37290.10 & 4.47 & 22.26 & $-52.7(-4.1)$ & $5.8(0.5)$ & $<16.4$ & $\ldots$ \\
\hline $4_{13}-3_{12}$ & 37954.95 & 7.60 & 20.87 & Blend & $\ldots$ & Blend & $\ldots$ \\
\hline $5_{15}-4_{14}$ & 45807.70 & 9.64 & 26.71 & $<9.5$ & $\ldots$ & $<9.5$ & $\ldots$ \\
\hline $5_{05}-4_{04}$ & 46602.80 & 6.71 & 27.82 & $-28.8(2.1)$ & $5.6(0.3)$ & $-23.4(1.7)$ & $4.0(0.4)$ \\
\hline $5_{14}-4_{13}$ & 47440.40 & 9.87 & 26.71 & Blend & $\ldots$ & $<9.2$ & $\ldots$ \\
\hline
\end{tabular}

Notes.

${ }^{a}$ Beam sizes, efficiencies, and continuum temperatures for each respective frequency can be found in Hollis et al. (2007).

${ }^{\mathrm{b}}$ Rest frequencies are all taken from Splatalogue; see Section 2 for complete references.

${ }^{c}$ The uncertainties for the intensities and line widths are Type B, $k=1(1 \sigma)$ (Taylor \& Kuyatt 1994).

${ }^{\mathrm{d}}$ Due to blended velocity components, line widths were shared during fitting.

${ }^{\mathrm{e}}$ Previously detected with the GBT with lower sensitivity and spectral resolution (Hollis et al. 2004b).

transition, where the velocity components were blended due to low signal-to-noise ratio and line widths were therefore fixed together during fitting, possibly artificially broadening the fit width.

\subsection{Propadienone}

No transitions of propadienone were identified in PRIMOS data. Of the known transitions between $4 \mathrm{GHz}$ and $50 \mathrm{GHz}$, only $a$-type transitions ( $\mu_{a}=2.156 \mathrm{D}, \mu_{b}=0.7914 \mathrm{D}$ ) (Brown et al. 1981) are predicted to be strong enough to be observed in PRIMOS data. It is important to note that the slight bend of the $\mathrm{C}_{3} \mathrm{O}$ chain in propadienone causes a small barrier, creating two tunneling states separated by a small $\left(0.1198 \mathrm{~cm}^{-1}\right)$ energy difference. $a$-type transitions occur within each substate while $b$-type transitions occur between the two tunneling states (Brown et al. 1987). $b$-type transitions within PRIMOS 


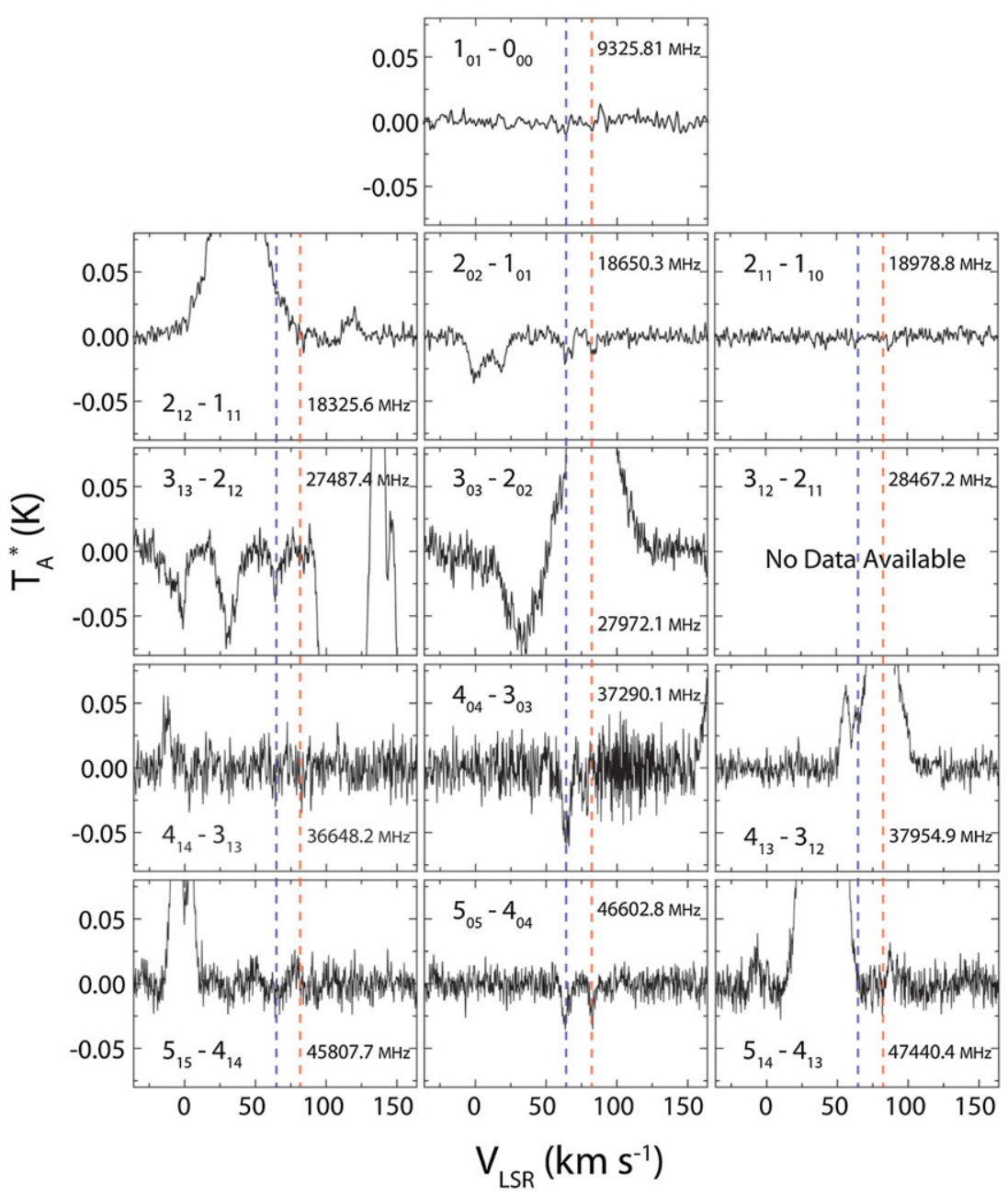

Figure 4. Observed propynal $a$-type transitions in PRIMOS data toward Sgr B2(N). Dashed vertical lines indicate the primary $+64 \mathrm{~km} \mathrm{~s}^{-1}$ component (blue) and the secondary $+82 \mathrm{~km} \mathrm{~s}^{-1}$ component (red).

frequency coverage are predicted to be significantly weaker due to a lower dipole moment and high upper state energies. Observations of all $a$-type transition rest frequencies within the PRIMOS coverage are presented in Figure 6 and upper limits on all $a$-type transition intensities are given in Table 3 .

\section{DISCUSSION}

Although our observational results support a detection of cyclopropenone and propynal and a non-detection of propadienone, it is important to additionally examine their abundance ratios. It may be possible for a given isomer to be more abundant, yet not detected, due to inherent line strengths or excitation issues. Using the formulation in Hollis et al. (2004a), and assuming a temperature of $15 \mathrm{~K}$, similar to other cold large organics detected with the PRIMOS survey, we calculate column densities of $\sim 10^{12} \mathrm{~cm}^{-2}$ for cyclopropenone, $\sim 10^{13} \mathrm{~cm}^{-2}$ for propynal, and an upper limit of $<10^{11} \mathrm{~cm}^{-2}$ for propadienone. Thus, if propadienone was actually equal in abundance to cyclopropenone or propynal, we would expect to see features 10-100 times the noise level, yet no such features are observed. These calculations assume continuum temperatures for Sgr B2(N) from Hollis et al. (2007), as well as LTE conditions. We wish to stress that our observations are not fit particularly well with these assumptions, suggesting that a large number of the observed lines are sub-thermally excited. If these features arise from non-LTE conditions, this may call into question our derived column densities, but would also invalidate the assumptions necessary for the MEP, strengthening our assertion that thermodynamically driven processes do not control the bulk of interstellar abundance ratios.

These results answer calls for further observational tests of the MEP from theoretical studies investigating the relative energies of multiple isomer families (Lovas et al. 2010; Karton \& Talbi 2014). In particular, Karton \& Talbi (2014) explicitly note that within the $\mathrm{C}_{3} \mathrm{H}_{2} \mathrm{O}$ isomer family, propadienone should be detected based on the predictions of the MEP and suggest further observational searches for the molecule. Its non-detection now leaves us with the question as to which synthesis routes result in the formation of cyclopropenone and propynal but not propadienone.

\subsection{Formation Chemistry on Grain Surfaces}

In the ISM, dust grains serve as catalysts for much of the rich organic chemistry that is observed (Herbst \& van Dishoeck 2009). Zhou et al. (2008) studied the non-equilibrium grain-ice chemistry in cold $(\sim 10 \mathrm{~K})$ regions of the ISM, finding that cyclopropenone is formed on grain surfaces via reactions between triplet $\mathrm{CO}$ and $\mathrm{C}_{2} \mathrm{H}_{2}$, i.e.,

$\mathrm{CO}+\mathrm{C}_{2} \mathrm{H}_{2} \rightarrow \mathrm{c}-\mathrm{C}_{3} \mathrm{H}_{2} \mathrm{O}$ 
Table 3

Observed Transitions of Propadienone

\begin{tabular}{|c|c|c|c|c|c|c|c|}
\hline \multirow{2}{*}{$\begin{array}{l}\text { Transition } \\
J_{k k}^{\prime}-J_{k k}^{\prime \prime}\end{array}$} & \multirow{2}{*}{$\begin{array}{c}\text { Frequency }{ }^{\mathrm{a}, \mathrm{b}} \\
(\mathrm{MHz})\end{array}$} & \multirow{2}{*}{$\begin{array}{l}E_{u} \\
(\mathrm{~K})\end{array}$} & \multirow{2}{*}{$\begin{array}{l}S_{i j} \mu^{2} \\
\left(\mathrm{D}^{2}\right)\end{array}$} & \multicolumn{2}{|c|}{$64 \mathrm{~km} \mathrm{~s}^{-1}$} & \multicolumn{2}{|c|}{$82 \mathrm{~km} \mathrm{~s}^{-1}$} \\
\hline & & & & $\Delta T_{A}^{*}(\mathrm{mK})$ & $\Delta V\left(\mathrm{~km} \mathrm{~s}^{-1}\right)$ & $\Delta T_{A}^{*}(\mathrm{mK})$ & $\Delta V\left(\mathrm{~km} \mathrm{~s}^{-1}\right)$ \\
\hline $1_{01}-0_{00}$ & 8645.16 & 0.41 & 4.65 & $<3.4$ & $\ldots$ & $<3.4$ & $\ldots$ \\
\hline $2_{12}-1_{11}$ & 17164.49 & 8.39 & 20.92 & No data & $\ldots$ & No data & $\ldots$ \\
\hline $2_{02}-1_{01}$ & 17290.18 & 1.24 & 9.30 & No data & $\ldots$ & No data & $\ldots$ \\
\hline $2_{11}-1_{10}$ & 17422.33 & 8.41 & 20.92 & No data & $\ldots$ & No data & $\ldots$ \\
\hline $3_{13}-2_{12}$ & 25746.59 & 9.63 & 37.19 & $<3.4$ & $\ldots$ & $<3.4$ & $\ldots$ \\
\hline $3_{03}-2_{02}$ & 25934.97 & 2.49 & 13.95 & $<3.6$ & $\ldots$ & $<3.6$ & $\ldots$ \\
\hline $3_{12}-2_{11}$ & 26133.34 & 9.66 & 37.19 & $<5.4$ & $\ldots$ & $<5.4$ & $\ldots$ \\
\hline $4_{14}-3_{13}$ & 34328.55 & 11.27 & 52.29 & $<3.3$ & $\ldots$ & $<3.3$ & $\ldots$ \\
\hline $4_{04}-3_{03}$ & 34579.38 & 4.15 & 18.60 & $<8.0$ & $\ldots$ & $<8.0$ & $\ldots$ \\
\hline $4_{13}-3_{12}$ & 34844.16 & 11.34 & 52.29 & $<9.2$ & $\ldots$ & $<9.2$ & $\ldots$ \\
\hline $5_{15}-4_{14}$ & 42910.28 & 13.33 & 66.93 & $<6.9$ & $\ldots$ & $<6.9$ & $\ldots$ \\
\hline $5_{05}-4_{04}$ & 43223.30 & 6.22 & 23.24 & $<8.6$ & $\ldots$ & $<8.6$ & $\ldots$ \\
\hline $5_{14}-4_{13}$ & 43554.72 & 13.43 & 66.94 & $<7.6$ & $\ldots$ & $<7.6$ & $\ldots$ \\
\hline
\end{tabular}

Notes.

${ }^{a}$ Beam sizes, efficiencies, and continuum temperatures for each respective frequency can be found in Hollis et al. (2007).

${ }^{\mathrm{b}}$ Rest frequencies are all taken from Splatalogue; see Section 2 for complete references.

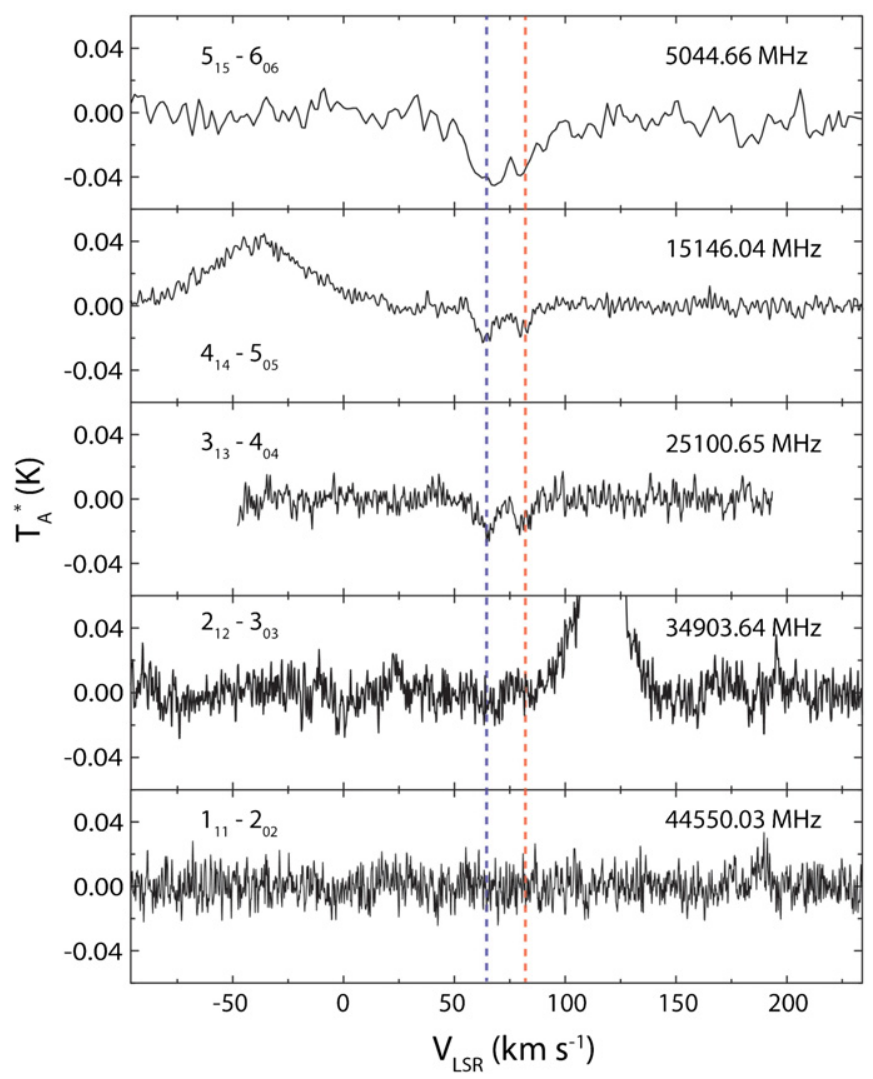

Figure 5. Observed propynal $b$-type transitions in PRIMOS data toward Sgr B2(N). Dashed vertical lines indicate the primary $+64 \mathrm{~km} \mathrm{~s}^{-1}$ component (blue) and the secondary $+82 \mathrm{~km} \mathrm{~s}^{-1}$ component (red).

and that propynal is formed in the bulk by carbon monoxide and acetylene through a radical pair reaction, i.e.,

$$
\mathrm{CO}+\mathrm{C}_{2} \mathrm{H}_{2} \rightarrow[\mathrm{HCO}-\mathrm{CCH}]^{*} \rightarrow \text { HCCCHO. }
$$

Zhou et al. (2008) found that cosmic ray protons $(\sim 10 \mathrm{MeV})$ are likely the source of energy that drives these reactions, inducing a $\mathrm{C}-\mathrm{H}$ cleavage in collisions with acetylene that initiates reactions (1) and (2). Experimental results from ultra-high vacuum irradiation of ice samples showed that the two metastable isomers of $\mathrm{C}_{3} \mathrm{H}_{2} \mathrm{O}$ were produced, with only a tentative detection of propadienone. The molecules formed in this way can then enrich the gas-phase abundance through sublimation, a particularly likely scenario in star-forming regions such as Sgr B2(N).

\subsection{Gas-phase Formation Chemistry}

In addition to formation pathways involving grain chemistry, there are several gas-phase reactions that are thought to produce cyclopropenone and propynal, but no feasible reactions have been proposed for propadienone production (Kwon et al. 2006; Petrie 1995; Irvine et al. 1988). A study by Quan \& Herbst (2007) attempted, in part, to reproduce the observed abundance of $\mathrm{c}-\mathrm{C}_{3} \mathrm{H}_{2} \mathrm{O}$ in $\mathrm{Sgr} \mathrm{B} 2(\mathrm{~N})$. Their network included the gas-phase formation pathway:

$$
\begin{gathered}
\mathrm{C}_{3} \mathrm{H}^{+}+\mathrm{H}_{2} \rightarrow \mathrm{c}-\mathrm{C}_{3} \mathrm{H}_{3}^{+}+h v \\
\mathrm{c}-\mathrm{C}_{3} \mathrm{H}_{3}^{+}+e^{-} \rightarrow \mathrm{c}-\mathrm{C}_{3} \mathrm{H}_{2}+\mathrm{H} \\
\mathrm{c}-\mathrm{C}_{3} \mathrm{H}_{2}+\mathrm{O}\left({ }^{3} P\right) \rightarrow \mathrm{c}-\mathrm{C}_{3} \mathrm{H}_{2} \mathrm{O} .
\end{gathered}
$$

Recently, the reactant $\mathrm{C}_{3} \mathrm{H}^{+}$in reaction (3) was detected in Sgr B2(N), suggesting the validity of this gas-phase formation route as a possible source of $\mathrm{C}_{3} \mathrm{H}_{2}$ (McGuire et al. 2013). The abundance of the cyclic form of $\mathrm{C}_{3} \mathrm{H}_{2}$ has been estimated to be 50-150 times greater than the linear form of $\mathrm{C}_{3} \mathrm{H}_{2}$ toward $\mathrm{Sgr} \mathrm{B} 2(\mathrm{~N})$ (Cernicharo et al. 1999), suggesting that it has the potential to be a parent molecule of c- $\mathrm{C}_{3} \mathrm{H}_{2} \mathrm{O}$ (Matthews \& Irvine 1985). The model of Quan \& Herbst (2007) was unable to reproduce the observed abundance of $\mathrm{c}-\mathrm{C}_{3} \mathrm{H}_{2} \mathrm{O}$ in $\mathrm{Sgr} \mathrm{B} 2(\mathrm{~N})$, but this is not too surprising, given that they did not include grain-surface reactions for the formation of cyclopropenone in their network.

\subsection{Interpretation of This Case Study and Conclusions}

From this collection of reactions, a picture emerges in Sgr B2(N) that there are simply more non-equilibrium formation pathways for propynal and cyclopropenone (gas and grain) 


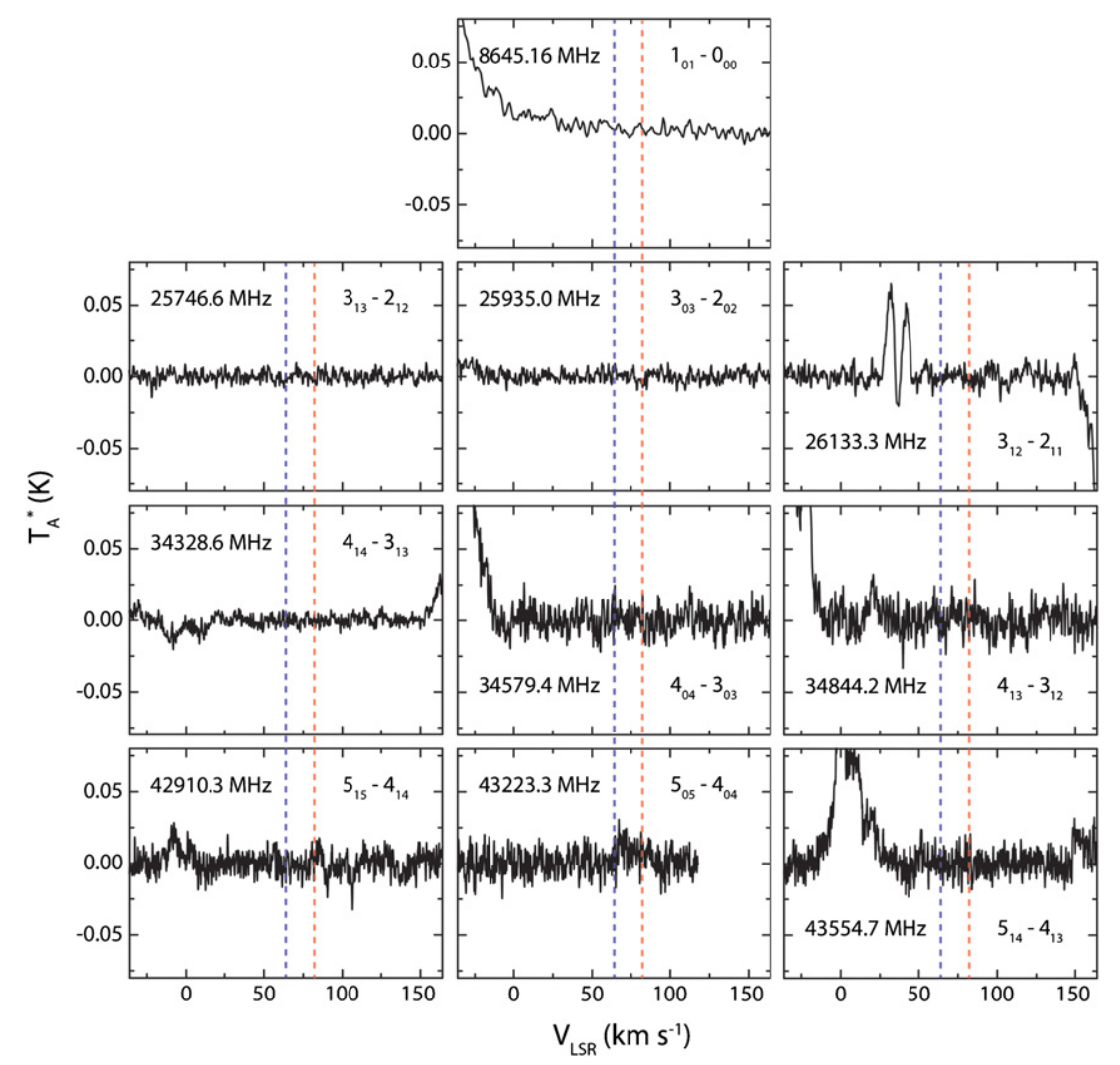

Figure 6. Observed propadienone transitions in PRIMOS data toward Sgr B2(N). Dashed vertical lines indicate the primary $+64 \mathrm{~km} \mathrm{~s}^{-1}$ component (blue) and the secondary $+82 \mathrm{~km} \mathrm{~s}^{-1}$ component (red).

than for propadienone, despite the fact that it is thermodynamically more stable. Additionally, Lovas et al. (2010) suggest that in grain-surface reactions, the surrounding ice matrix may quench excess energy, preventing rearrangement to more thermodynamically favorable species such as propadienone. These results are not limited to our current work, however; previous studies of other isomer systems such as $\mathrm{HNC} / \mathrm{HCN}$ have also suggested the importance of kinetic control in determining abundance ratios.

The ground state energy of the metastable HNC is $7240 \mathrm{~K}$ above HCN (Bowman et al. 1993). Thus, if Lattelais et al. (2009) were correct in stating that relative isomeric abundances can be explained in terms of ground state energies, one would expect $\mathrm{HCN}$ to be much more abundant than HNC. However, observations by Schilke et al. (1992) of OMC-1 showed an HNC/HCN ratio of almost unity. Further observations (Padovani et al. 2011; Hébrard et al. 2012; Aalto et al. 2012) demonstrate that as temperatures decrease, the $\mathrm{HNC} / \mathrm{HCN}$ ratio increases. Studies by Herbst et al. (2000) and Graninger et al. (2014) indicate that the relative abundances of $\mathrm{HCN}$ and $\mathrm{HNC}$ can best be understood in terms of kinetic control, specifically that certain destruction pathways for $\mathrm{HNC}$ involving neutral-neutral reactions with $\mathrm{H}$ do not occur at low temperatures due to energy barriers.

We therefore conclude that the MEP does not have substantial predictive power for determining the most likely isomer to be observed in a given isomer family, especially in blind application with no prior knowledge. This suggests that as next generation capabilities become available, specific emphasis should be placed on investigating molecules and molecular reactions under kinetic control, especially when investigating regions where LTE assumptions are unlikely to hold. With a better understanding of chemical environments, formation and destruction mechanisms, and excitation conditions, it may be possible to apply a modified version of the MEP for certain isomer families in specific environments, but further observational and laboratory work is necessary for rigorous predictions.

R.A.L. gratefully acknowledges funding from an NSF Graduate Research Fellowship and the Virginia Space Grant Program, as well as support from the College Science Scholars program at the University of Virginia. B.A.M. gratefully acknowledges funding from an NSF Graduate Research Fellowship, and G. A. Blake for his support. We thank an anonymous referee for important comments. The National Radio Astronomy Observatory is a facility of the National Science Foundation operated under cooperative agreement by Associated Universities, Inc.

\section{REFERENCES}

Aalto, S., Garcia-Burillo, S., Muller, S., et al. 2012, A\&A, 537, A44 Belloche, A., Müller, H. S. P., Menten, K. M., Schilke, P., \& Comito, C. 2013, A\&A, 559, A47

Benson, R. C., Flygare, W. H., Oda, M., \& Breslow, R. 1973, JAChS, 95, 2772 Bowman, J. M., Gazdy, B., Bentley, J. A., Lee, T. J., \& Dateo, C. E. 1993, JChPh, 99, 308

Brown, R. D., \& Godfrey, P. D. 1984, AJCh, 37, 1951

Brown, R. D., Godfrey, P. D., \& Champion, R. 1987, JMoSp, 123, 93

Brown, R. D., Godfrey, P. D., Champion, R., \& McNaughton, D. 1981, JAChS, 103,5711

Cernicharo, J., Cox, P., Fossé, D., \& Güsten, R. 1999, A\&A, 351, 341

Crockett, N. R., Bergin, E. A., Neill, J. L., et al. 2014, ApJ, 787, 112

Faure, A., Remijan, A. J., Szalewicz, K., \& Wiesenfeld, L. 2014, ApJ, 783, 72 Garrod, R. T. 2013, ApJ, 778, 158

Garrod, R. T., Weaver, S. L. W., \& Herbst, E. 2008, ApJ, 682, 283

Graninger, D. M., Herbst, E., Öberg, K. I., \& Vasyunin, A. I. 2014, ApJ, 787,74 
Hébrard, E., Dobrijevic, M., Loison, J. C., Bergeat, A., \& Hickson, K. M. 2012, A\&A, 541, A21

Herbst, E., Terzieva, R., \& Talbi, D. 2000, MNRAS, 311, 869

Herbst, E., \& van Dishoeck, E. F. 2009, ARA\&A, 47, 427

Hocking, W. H., Gerry, M. C. L., \& Winnewisser, G. 1974, ApJL, 187, L89

Hollis, J. M., Jewell, P. R., Lovas, F. J., \& Remijan, A. 2004a, ApJL, 613, L45

Hollis, J. M., Jewell, P. R., Lovas, F. J., Remijan, A., \& Møllendal, H. 2004b, ApJL, 610, L21

Hollis, J. M., Jewell, P. R., Remijan, A. J., \& Lovas, F. J. 2007, ApJL, 660, L125

Hollis, J. M., Remijan, A. J., Jewell, P. R., \& Lovas, F. J. 2006, ApJ, 642, 933

Irvine, W. M., Brown, R. D., Cragg, D. M., et al. 1988, ApJL, 335, L89

Karton, A., \& Talbi, D. 2014, CP, 436, 22

Kwon, L.-K., Nam, M.-J., Youn, S.-E., et al. 2006, JChPh, 124, 204320

Laas, J. C., Hays, B. M., \& Widicus Weaver, S. L. 2013, JPCA, 117, 9548

Lattelais, M., Bertin, M., Mokrane, H., et al. 2011, A\&A, 532, A12

Lattelais, M., Pauzat, F., Ellinger, Y., \& Ceccarelli, C. 2009, ApJL, 696, L133

Lattelais, M., Pauzat, F., Ellinger, Y., \& Ceccarelli, C. 2010, A\&A, 519, A30

Loomis, R. A., Zaleski, D. P., Steber, A. L., et al. 2013, ApJL, 765, L9

Lovas, F. J., Plusquellic, D. F., Widicus Weaver, S. L., McGuire, B. A., \& Blake, G. A. 2010, JMoSp, 264, 10

Matthews, H. E., \& Irvine, W. M. 1985, ApJL, 298, L61

McGuire, B. A., Carroll, P. B., Loomis, R. A., et al. 2013, ApJ, 774, 56
Müller, H. S. P., Schlöder, F., Stutzki, J., \& Winnewisser, G. 2005, JMoSt, 742,215

Müller, H. S. P., Thorwirth, S., Roth, D. A., \& Winnewisser, G. 2001, A\&A, 370, L49

Neill, J. L., Bergin, E. A., Lis, D. C., et al. 2014, ApJ, 789, 8

Neill, J. L., Muckle, M. T., Zaleski, D. P., et al. 2012, ApJ, 755, 153

Padovani, M., Walmsley, C. M., Tafalla, M., Hily-Blant, P., \& Pineau Des Forêts, G. 2011, A\&A, 534, A77

Pearson, J. C., Sastry, K. V. L. N., Herbst, E., \& De Lucia, F. C. 1997, ApJ, 480, 420

Petrie, S. 1995, ApJL, 454, L165

Pickett, H. M., Poynter, R. L., Cohen, E. A., et al. 1998, JQSRT, 60, 883

Quan, D., \& Herbst, E. 2007, A\&A, 474, 521

Remijan, A. J., Hollis, J. M., Lovas, F. J., et al. 2008, ApJL, 675, L85

Remijan, A. J., Markwick-Kemper, A., \& ALMA., (Working Group on Spectral Line Frequencies) 2007, BAAS, 39, 132.11

Sarrasin, E., Abdallah, D. B., Wernli, M., et al. 2010, MNRAS, 404, 518

Schilke, P., Walmsley, C. M., Pineau Des Forets, G., et al. 1992, A\&A, 256, 595

Snyder, L. E., \& Buhl, D. 1971, ApJL, 163, L47

Taylor, B. N., \& Kuyatt, C. E. 1994, NIST Tech. Note 1297 (Washington, DC: US Government Printing Office)

Turner, B. E., \& Apponi, A. J. 2001, ApJL, 561, L207

Ulich, B. L., \& Haas, R. W. 1976, ApJS, 30, 247

Winnewisser, G. 1973, JMoSp, 46, 16

Zaleski, D. P., Seifert, N. A., Steber, A. L., et al. 2013, ApJL, 765, L10

Zhou, L., Kaiser, R. I., Gao, L. G., et al. 2008, ApJ, 686, 1493 